\title{
MOBILE TELEMEDICINE FOR DIABETES CARE
}

\author{
Iñaki Martínez-Sarriegui ${ }^{1.3}$, Gema García Sáez ${ }^{1.3}$, M . Elena Hemando ${ }^{1.3}$, \\ Mercedes Rigla ${ }^{2,3}$, Eulalia Brugués ${ }^{2,3}$, Alberto de Leiva ${ }^{2.3}$, Enrique J. Gómez ${ }^{1.3}$ \\ 'Bioengineering and Telemedicine Group \\ Technical University of Madrid \\ Ciudad Universitaria, 28040 Madrid, Spain \\ Endocrinology Department \\ Hospital Sant Pau \\ Mas de Casanovas 90, 08025 Barcelona, Spain \\ ${ }^{3}$ CIBER-BBN: Bioengineering, Biomaterials and Nanomedicine Networking \\ Research Centre \\ Instituto de Salud Carlos $1 \mathrm{H}$, \\ Sinesio Delgado 6, 28029 Madrid, Spain \\ E-mail: imartinez@gbt.tfo.upm.es
}

Diabetes Mellitus is nowadays one of the most frequent non-contagious diseases in the world and remains a major health problem for the national health care programs. It is well proved that Telemedicine helps diabetic patients controlling their glucose levels. facilitating their dayto-day therapy management and the communication with health care personnel. The rapid growth and development of information technologies in the areas of mobile computing and mobile Intemet is shaping a new technological scenario of telemedicine and shared care systems. In this chapter we will show one approach to Mobile Telemedicine for Diabetes Care.

\section{Introduction}

According the International Diabetes Federation ${ }^{1} 5.1 \%$ of the world population suffers from diabetes and the forecast for 2025 is that it will increase to the $6.3 \%$. In Western societies the numbers rise to the $7.8 \%$ nowadays and $9.5 \%$ by 2025 . The situation of diabetes makes heavier 
due to the number of people with alteration of glucose tolerance that affects to the $8.2 \%$ of the world population and by 2025 it will affect to the $9 \%$. Another alarming fact is that for each case of diagnosed diabetes there is another that is not diagnosed.

Diabetes remains a major health problem being responsible of up to $8 \%$ of national health care expenditure ${ }^{2}$ Diabetes Mellitus is a chronic disease characterised by a sustained elevated blood glucose level, caused by a reduction in the action of insulin secretion where related metabolic disturbances generate severe, acute and long-term complications that are responsible for premature death and disability ${ }^{3}$. The World Health Organization projects that diabetes deaths will increase by more than $50 \%$ in the next 10 years without urgent action ${ }^{4}$. Most notably, diabetes deaths are projected to increase by over $80 \%$ in upper-middle income countries between 2006 and 2015 .

Due to its multifactorial and systemic character, diabetes mellitus has been considered a paradigm of chronic disorders which has led to an extensive application of information technologies in diabetes care Nowadays telemedicine provides an integrated approach to information technology tools, which enhances co-operation between users, information and knowledge sharing.

Over the last three decades, diabetes has been a mayor focus for biomedical engineering efforts to improve the diagnosis, monitoring and treatment of diabetic people. Earlier experiences aimed to facilitate the remote monitoring of patients from home by the transmission of computerised blood glucose profiles to the hospital ${ }^{6,7,8}$. Most of interactive telemedicine services were delivered using a distributed approach integrating "Patient Units" (PU), implemented on a personal computer or a palmtop and used by patients during their daily living; and "Medical Workstations" (MW), used by physicians and nurses at the hospital $^{9.10,11,12}$. Technology evolution has enabled the development of advanced systems based on more powerful, portable and easy to use terminals and applications, such as hand-held electronic diaries with touch-screen ${ }^{13}$, video telephones ${ }^{14}$, or web-based prototype systems $s^{9,15}$.

The rapid growth and development of information technologies during recent years in the areas of mobile computing, computer-telephony integration (CTI) and mobile Internet is changing the way people access 
to common services. Today people can buy cinema tickets, consult the traffic situation, pay their bills and many other actions with their mobile telephone or PDA. And a growing sector of citizens demands more services available in a mobile way.

As more and more people use mobile handsets, a revolution is taking place in computing and telecommunications. Two extraordinary industries -the Internet and mobile communications- are converging. But this is just the beginning. As the third industry -consumer electronicsand the fourth -media and entertainment- join in changes in consumer markets are inevitable, as evidenced by the explosive growth of mobile media, games and entertainment ${ }^{16}$.

Telemedicine and shared care systems should not obviate the technology and market evolution. Today the seamless integration of available mobile and wireless technologies allows building a new scenario for telemedicine and shared care in diabetes in which the traditional concepts of PU and $\mathrm{MU}$ are blurred, suffering a profound transformation evolving to a new multi-access, mobile, universal and ubiquitous workspace concept for diabetes care ${ }^{17}$.

In this chapter we present the telemedicine services requisites and the technical requirements of mobile telemedicine systems for diabetes care. These considerations were taken into account to build a multi-access mobile telemedicine workspace, implemented over the sustenance of a multi-agent architecture. This workspace provides universal access to a wide range of services both for patients and professionals. The chapter finishes drawing some conclusions about the current telemedicine and shared care experiences and the possibilities of mobile technologies in diabetes care in the future.

\section{The Diabetes Care challenge}

\subsection{The medical problem}

Diabetes Mellitus is a group of metabolic diseases characterised by a sustained elevated blood glucose level (hyperglycemia), caused by defects in insulin secretion, insulin action or both. Insulin facilitates entry 
of glucose into muscle, adipose and several other tissues and stimulates the liver to store glucose in the form of glycogen. The chronic hyperglycemia generates severe, acute and long-term complications that are responsible for premature death and disability ${ }^{3}$. The cause of diabetes continues to be a mystery, although both genetics and environmental factors such as obesity and lack of exercise appear to play roles ${ }^{18}$.

The vast majority of cases of diabetes fall into three broad categories: Type 1 , Type 2 and gestational diabetes ${ }^{19}$. Type 1 diabetes comprises $10 \%$ of all cases with diabetes. The cause is an absolute deficiency of insulin secretion due to beta cells destruction, and the glucose cannot be metabolized. People with type I diabetes need insulin administration to use glucose from meals. By taking a good control of blood glucose levels, type 1 diabetic patients can expect to have an almost normal life span as the benefits of intensive therapy management have been well established and include reduced long-term complications ${ }^{20}$.

Type 2 is the most prevalent form of diabetes, comprising $90 \%$ of diabetes cases. It results from a combination of resistance to insulin action and an inadequate compensatory insulin secretor response. People with type 2 need diabetes oral medication or insulin injections to control their blood glucose levels. The inability of a large proportion of the population in the more affluent western societies to cope with the excess caloric supply together with a lack of physical exercise results in a greater prevalence of Type 2 diabetes.

Gestational diabetes may give rise to several adverse outcomes, including congenital malformations, increased birth weight and an elevated risk of prenatal mortality and affects about $4 \%$ of all pregnant women. Strict metabolic control may reduce these risks to the level of those of non-diabetic expectant mothers.

\subsection{Diabetes Treatment}

Effective control of patients' blood glucose level minimizes the progression of the disease and reduces the risk of long-term neurological, renal and cardiovascular complications. The treatment of diabetic patients attempts to achieve normoglycemia by maintaining a careful balance between diet, physical exercise and insulin therapy. 
Patients monitor their own blood glucose levels (self-monitoring) during the daily life to predict and avoid hypoglycemia (low blood sugar level) and hyperglycemia (high blood glucose level) and to take decisions regarding the adjustment of changes of insulin doses, meals and physical activity.

Insulin therapy aims to mimic the physiological insulin patterns delivered by the pancreas in non-diabetic people with the injection of short-acting insulin given under the skin (subcutaneously) just before the meal and long-acting insulin given in the morning and evening to simulate the basal supply.

Insulin cannot be orally taken as it would be broken down during digestion. Therefore, insulin must be injected and its efficiency depends on when glucose from the taken food starts to enter your blood ${ }^{18}$ and in what place of the body is injected.

Scientific prospective studies have provided evidence on the use of intensive insulin therapy, based on multiple insulin injections or continuous insulin delivering, as the most appropriate method to equilibrate patient's blood glucose and to delay the onset and slow the progression of complications later in life.

To obtain the best care outcomes, diabetic people should receive medical care from a physician-coordinated team ${ }^{21}$. Such teams may include physicians, nurses, dieticians, pharmacists and mental health professionals with expertise and interest in diabetes. They assess the patients' glycemic control on the basis of patient reported monitoring blood glucose data as well as by the glycated hemoglobin (HbAlc) test, which provides an indication of the average blood glucose level over the previous 2 to 3 months. This collaborative and integrated shared care approach requires that individuals with diabetes assume an active role in their care requiring in many cases a better patient empowerment and education.

However the fulfilment of the current guidelines in diabetes management ${ }^{22}$ implies a significant increase in the amount of patient data to be monitored, increasing physicians and nurses" workload and raising immediate health care costs. 


\subsection{Telemedicine and Shared Care Services in Diabetes Management}

A telemedicine system for chronic care has to help physicians' decision making. In the case of diabetes mellitus the responsibility of data collection is shared between patients, who record data during daily life (self-monitoring data) and the healthcare persomnel, who capture data during hospital visits (hospital patient record) This suppose a huge quantity of data and increases physicians' workload analyzing them in order to take therapeutic decisions. The telemedicine system must provide enough information to enable assessment of the patient's condition and must present the relevant patient clinical data to define a therapeutic change. It should analyse patient's data, filtering the information, and generate automatic intelligent alarms to focus doctors' attention on those points where non-desired deviations in the metabolic control are found.

A telemedicine system for diabetic people has to provide Telemonitoring of the patients' monitoring data in order to allow physicians knowing the state of the patient. It should also allow Teleconsultation between patients and doctors through an asynchronous message exchange. Another service that it must supply is the Supervised Care, providing patients with a "supervised autonomy". Patients carry out day-to-day therapy changes and send this information to the physician who reviews the data and validates patients decisions or proposes further adjustments of the treatment.

To obtain the best care outcomes, diabetic people should receive medical care from a physician-coordinated team. The information technologies provide with helpful tools for this coordination. One example is the application of Computer Supported Collaborative Work (CSCW) current theories and tools to create new information technologies and telecommunication workspaces to bear diabetes shared care ${ }^{23}$.

The services to be provided by a shared care workspace for diabetes management are: Multi-channel messaging services, to allow users to send and receive messages in any format despite of the original message's format; Shared information dissemination and request, to send data automatically to users interested on them using both pull and push methods; Group and activity cowareness: to integrate an event and 
activities notification service -the aim of this service is to aware every user of the most relevant events occurring at the lowest cost-; Shared agenda (scheduling meeting/visits), this facility let users to share an agenda complementing the above services.

\subsection{Ambulatory artificial pancreas}

The combination of insulin pumps and continuous glucose monitoring systems seems to be the best current solution to achieve a good metabolic control for insulin-dependent diabetic people. Continuous glucose monitoring can detect glycemia patterns that cannot be discovered only by means of few diary measurements ${ }^{24}$. The possibility to obtain a complete blood glucose profile allows monitoring the suitability of an intensive therapy and can be used to fine-tune the therapy. The old concept of the "artificial pancreas" is starting to be a reality supported by the availability of these technologies and the integration of control systems able to close the loop modifying the pump parameters ${ }^{25}$.

A closed-loop device capable of maintaining normoglycemia over extended periods of time could dramatically improve the quality of metabolic control of insulin-dependent diabetic patients ${ }^{26}$. An artificial pancreas contains three primary components: 1) An insulin pump; 2) A continuous glucose sensor; and 3) A closed-loop mathematical algorithm to regulate the pump given a sensor measurement.

Over the last decades, the development of an artificial pancreas has been a huge challenge on the application of biomedical technologies to diabetes therapy. The evolution of continuous glucose monitoring and insulin pumps technologies is creating a very promising situation in the next future. However, the current reliability constraints of continuous glucose sensor, the non-linearity of the glucoregulatory system and the inherent complexity of the design of a glucose controller for a SC-SC ( $\mathrm{SC}$, subcutaneous) setup are still some of the problems to be faced before obtaining a portable artificial pancreas. 


\section{Technical Requirements of Mobile Telemedicine Systems for Diabetes Care}

We have seen some of the requirements of Telemedicine and Shared Care Systems for diabetes care. These requirements are general but for mobile systems there are some considerations and other requirements that we should consider.

The applications that are supposed to rule on a mobile device, PDA or mobile telephone, have to take into account the following considerations $^{27.28,29}$ :

- Concise content: Only show relevant information using understandable abbreviations.

- Current process recuperation: The user's task could be interrupted by a phone call or a loss of coverage. The application must recover the process after the interruption.

- Writing options: Include all predictable texts in order to avoid user inserting it because of the text input limitations of mobile devices.

- Novigation: It is recommended that the application has a lot of categories with low depth. The information introduced should be stored in order to guarantee backward navigation.

- Reduce the number of keystrokes: Especially in mobile telephones where the text input is made with several keystrokes for each letter.

- Velocity: When the application makes use of Internet it has to be considered that the user pays for time or transmitted bytes so the connection should be fast enough.

- Device limitations: Mobile devices have limitations of battery, velocity of process, storage capacity and graphic. These limitations must be considered at the design phase.

- Easy-to-use interface: The system should provide simple interfaces.

- Media adaptability: The system should provide support for different data: images, video and text. 
- Modular design: The system should have a modular design so that it allows for the development of a roadmap for growth that can accommodate future generations of functionality

The implementation of an Ambulatory Artificial Pancreas requires additionally features:

- Always-on technology: The closed loop algorithms have to run 24 hours a day, reading continuous glucose devices and operating insulin pumps in real time. Robust devices and long duration batteries are crucial for this critical task.

- Services for remote supervision. PDA's applications usually work as client processes unable to provide information to external agents. The artificial pancreas application requires to include server functionalities (e.g. RPC or distributed CORBA objects) to allow remote supervision and even remote operation of medical devices.

Moreover, any mobile telemedicine application must fulfill some other requirements:

- Security: Health care data are protected by official privacy laws and data protection. Therefore mobile telemedicine application must be very careful with the security at all levels: communication, storage...

- Communication with medical devices: This communication can be wireless (i.e.Bluetooth, IrDA, WiFi) or by cable. In any case the security and integrity of the data transferred from/to the medical device should be guaranteed.

- Controllability: The system should support remote control functions. The healthcare provider should be empowered with the ability to control the media content according to medical specialties or his/her personal preference. For example, the health provider is allowed to control ECG sample rate, video frame rate, and image quality, etc. 


\section{Building the Mobile Telemedicine System}

Actually there are different Mobile Telemedicine applications for Diabetes Care. Some mobile companies are commercializing mobile solutions that allow patients download data to a mobile telephone from a glucometer using a cable ${ }^{30}$, via Bluetooth ${ }^{31}, \operatorname{IrDA}^{32}$. Besides, some other systems download data from the glucometer to a private network via a long range wireless connection ${ }^{33}$.

Patients and doctors can access to the data in the network using a web based application or a mobile telephone application. Some applications provide more facilities like manual data recollection or graphic visualization of previous data in addition to data analysis and alarm generation ${ }^{34}$.

There are some non-commercial experiences that allow glucometer data downloading ${ }^{35,36}$. Another mobile application for diabetes care uses mobile telephone to develop text messages with specific behavioral health strategies to young diabetes patients ${ }^{37}$.

In this section we are going to talk about the DIABTel Telemedicine System for Type I diabetic patients developed by the authors. DIABTel is a multi-access mobile telemedicine workspace, implemented over the sustenance of a multi-agent architecture. This workspace provides universal access to a wide range of services both for patients and professionals.

\subsection{The DIABTel distributed architecture}

The complexity and flexibility required to build the mobile workspace is achieved with the definition of a middleware multi-agent architecture that comprises a full range of non-expensive and widely accepted information technologies offering to users a universal, easy-to-use, online and cost-effective access to telemedicine and information services. This architecture allows users to access the information whatever access terminal or combination of terminals they choose. It facilitates the use of heterogeneous software and/or hardware solutions making possible that each Health Care Organization configure the number and the kind of services they want to offer to their own users. Moreover, it creates a 
continuous record of the users' actions to monitor all the collaboration processes involved in shared care to improve the quality of care.

This integrated platform embeds several technical implementations to allow the access to a wider range of services both for patients and for professionals: consultation of patients' records; text and voice mailing; printing of reports; alarm management; visit management; telecare; teleeducation and intelligent therapy advising.

The architecture yields on three components: the Agents, the multiaccess Organizer and the database. The agents collaborate to guarantee a homogeneous access of the users to the system services allowing users to access the information whatever access terminal or combination of terminals they choose. The number of agents is not limited initially and it only depends on the kind of terminals that will be used at each site.

There are two types of agents:

- Communication Server agents are in charge of communications with the different user terminals. Each communication agent is in charge of managing the communication process between the System and one specific user terminal. Their main functionality is to receive data from users updating it into the database and to retrieve data from the database to be presented at the user's terminal. They have the responsibility of performing the security policies for user access control, data confidentiality and data integrity during data transfers.

- Application Server agents: in charge of data analysis and data processing.

The Multi-access Organizer is a message-oriented middleware module that is in charge of coordinating the interoperability between all the agents integrated into the architecture. The communication between the Organizer and the agents is done using "event messages" encapsulated into TCP/IP messages. The Organizer receives a message from each agent whenever an event occurs and activates the next action to be done. Additionally the Organizer includes: Event Registry Services that enable the establishment of an event registry and event monitoring services and Smart Routing Services that ensure the message gets delivered to the appropriate recipients in the correct sequence. 


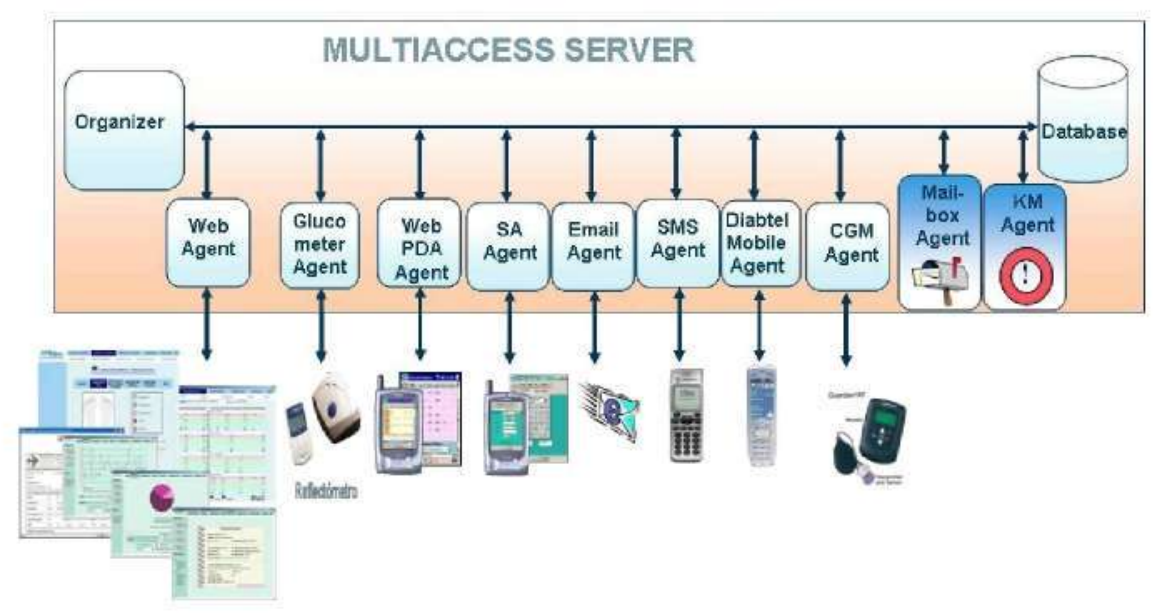

Fig. 1. The mobile multi-access telemedicine workspace

The number and nature of the events is not fixed and can be defined at any system implementation according to their preferences or specific care protocols. During the exploitation of the system, the multi-agent architecture allows the inclusion of new events without affecting previous service performance. Likewise, the action the Organizer triggers when a certain event is received is fully configurable.

The benefit of the multi-access multiagent architecture is that it isolates the different communication processes from the rest of the system, so if some communication server needs to change its communication protocol the system is not affected and only this server has to be modified. The definition of independent agents allow each site to have its own preferred communication servers working and, even more, the addition of new communication servers is transparent to the existing ones.

\subsection{Mobile applications in DIABTel system}

Users can interact with the common workspace using several terminals such as Web, PDAs and mobile phones. The user applications running on these terminals have a set of common functions both for patients and the 
health care professionals that enable the provision of the diabetes management services described above.

The main access method to reach the information in the multi-access system is the web graphical interface, offering the complete functionality of the telemedicine/shared care services with minimal human factors limitations. Through the DIABTel web access the users, patients and doctor, can consult the electronic patient logbook, insert new data, consult or create new treatments, access to cooperative electronic mail, download data from glucometer, see graphical information: clinical as much as system use information, etc.

The 'mobile applications' implements some of these functionalities, adapting them to the limitations of the mobile devices and following the considerations exposed in 3.2 .

\subsubsection{PDA Smart Assistant application}

The Smart Assistant (SA) application configures one of the most advanced approaches to mobile telemedicine close-loop systems in diabetes management ${ }^{38}$. It provides patients with several closed-loop control strategies (personal and remote) that offer an augmented information self-management environment to increase patient empowerment. The SA has an independent executable algorithm implemented, based on a non-linear MPC with Bayesian learning. The algorithm allows real-time control of the insulin pump based on glucose data. It needs also the meal intakes and patient's personal data. The algorithm can work in a continuous mode, providing calculation for basal insulin rates every 15 minutes.

The PDA application is a portable and mobile solution which provides the patients with flexible tools for data visualisation and with local and remote communication services. The Smart Assistant is able to manage a local database, allowing the patient to work isolated from the telemedicine server without the need of a permanent active communication link. On demand by the user, the Patient Unit synchronizes the local database with the server database causing the automatic updating of all the new information in both directions. 


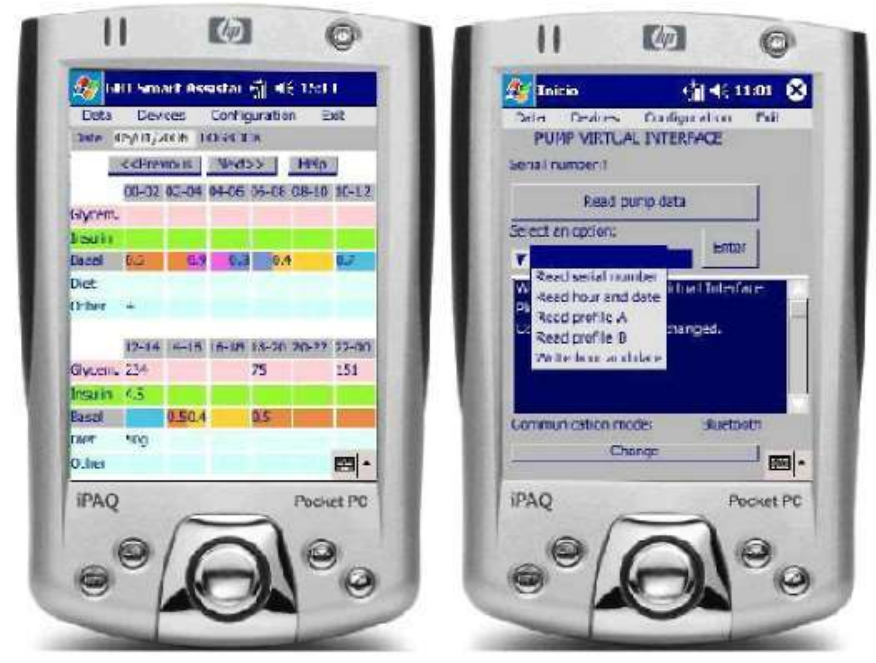

Fig. 2. The 'logbook' and pump communication scenarios for the PDA application and iPAQ h2210 PDA

The most characteristic functionality developed for the PDA application is the "patient electronic logbook" where the patient could perform the following tasks:

- Manage hisher monitoring data. It implies visualization and edition of the patient logbook. It is possible to select the period or the single time slice for the data to be introduced or visualized. The patient can visualize or insert meal data, glucose data, insulin data, device events (needle change, catheter change, etc.), individual activities (exercise), additional data (medication, stress, etc.)

- Visualization of graphics and statistical data analyses. Three types of graphics are available: plot, pie or temporal.

- Consult his/her active therapy. The last therapy (insulin and diet) proposed by the physician is updated in the application after each data synchronization.

- To download data from the insulin pump or the glucometer. The communication with the insulin pump is wireless (Bluetooth or IrDA) and with the glucometer is with a serial cable. 
The PDA application can be configured by the patients. Some of the options of configuration are preferred language, preferred blood glucose units ( $\mathrm{mg} / \mathrm{dl}$ or $\mathrm{mmol} / \mathrm{l}$ ) for the visualization scenarios, preferred starting hour for the logbook representation. It is also possible to enter additional patient information (weight, date of birth, age, birth date, etc.) It also allows introducing authentication data of the patient that uses the application, such as login and password and modifying the configuration settings for each patient.

The PDA application is developed in Java in order to be portable on multiple platforms. The portability of the application has been successfully tested in conventional PCs and in the PDAs iPAQ hp2210 and TSM500, both with Windows CE operating system. It's necessary to install CrEme v3.24 JVM technology for embedded platforms in order to run the application. The TSM500 has GPRS mobile connection integrated and for the iPAQ the AudioVox RTM 8000 card was used to provide the GPRS communication capabilities.

The Smart Assistant was evaluated in a clinical experiment and demonstrated an improvement in the glycemic control in pump treated patients with Type 1 diabetes ${ }^{39}$.

\subsubsection{WebPDA}

The WebPDA is a Web application developed specifically designed taking into account the PDA limitations. The DIABTel Web application is optimized for a high-resolution display $(1024 \times 768)$ in order to present as much information as possible. When accessing with a lower resolution device scrolling is allowed, but the PDA resolution is much lower so too much scrolling is necessary to access to Web application. The ' $y$ ' dimension in PC screens is bigger than the ' $x$ ' dimension, but in PDAs is opposite. So a Web application could not be seen properly in a PDA screen.

The WebPDA is a Web application developed specifically designed taking into account these PDA limitations. It can be considered a lighter 
version from the Web application. The functionalities of the WebPDA application are:

- Doctors: Patient selection, Creation and visualization of treatments, Electronic logbook visualization, Visualization and creation of electronic mails, Visualization and creation of news.

- Patients: Electronic logbook visualization, Visualization of treatments, Visualization of news, Visualization and creation of electronic mails.
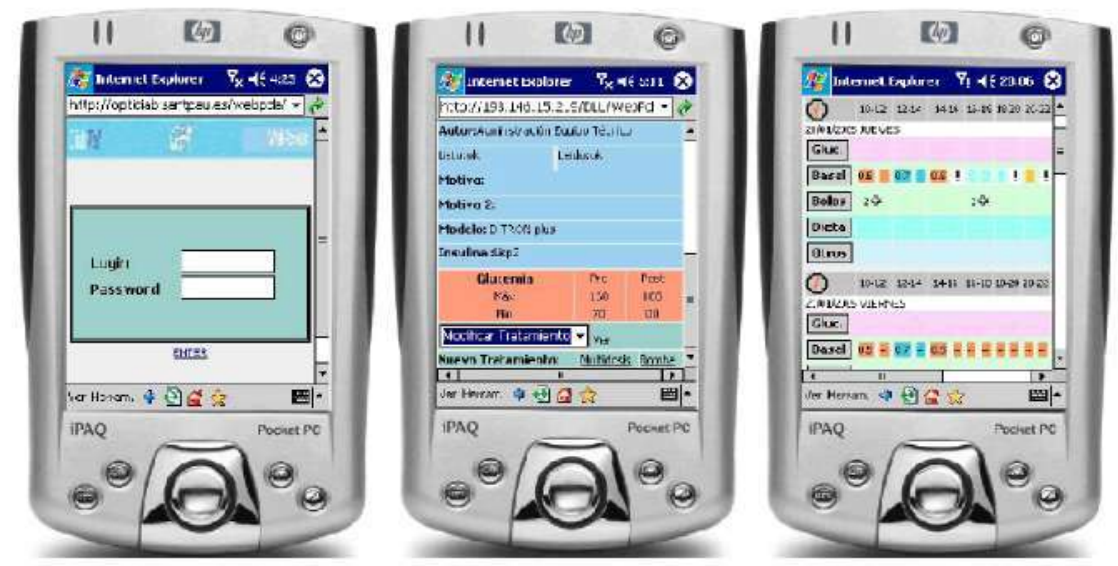

Fig. 3. The WebPDA application

\subsubsection{DIABTelMobile}

DIABTelMobile is a mobile telephone application. The functionalities are: Introduction, storing, sending and visualization of patient monitoring data, visualization of treatment, transmition and reception of messages and data downloading from glucometer via bluetooh.

The DIABTelMobile has been developed in Java Microedition, J2ME, using the CLDC 1.0 configuration and the MIDP 2.0 specification. Theoretically, it can work in any mobile telephone that implements the MIDP 2.0 specification and also the Bluetooth JSR82 specification. But the practice shows that not all the mobile telephones that are supposed to implement the MIDP 2.0 and the JSR82 specifications make it wholly. 
The application was tested in the TSM520 mobile telephone with Windows Mobile Operating System.
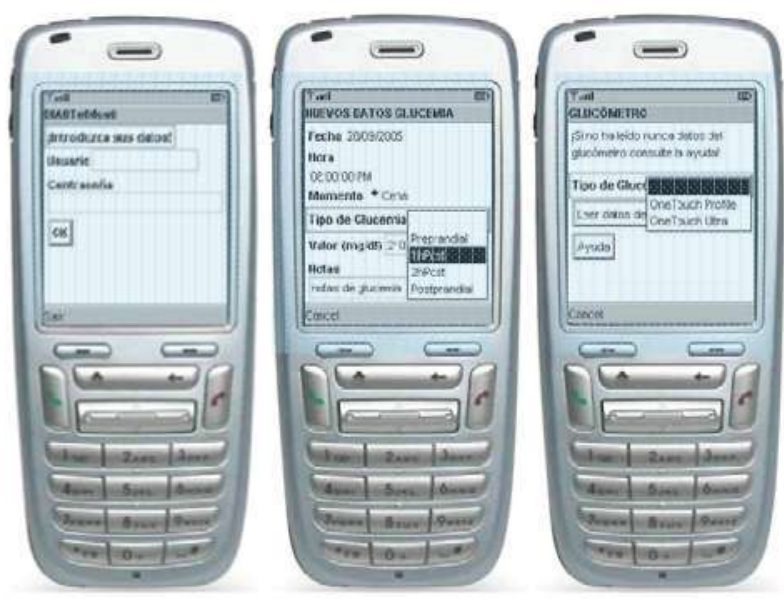

Fig. 4. The DIABTelMobile application

\section{Conclusions}

Wireless technologies and the further integration of medical devices into mobile scenarios are the pillars for new mobile services that operate in the personal area network optimising the patient intervention for data retrieval and communication.

Moving in this direction, mobile telemedicine increases the quality and the quantity of the information collected by patients, improving the physician-patient communication and enhancing the decision-making process to achieve effective therapy adjustments in a collaborative and supervised way.

The implementation of these mobile services requires the definition of distributed architectures including strict strategies for security to guarantee patient privacy, data confidentiality and integrity and auditing of the clinical acts are mandatory. The limitations of mobile devices, such as data processing and batteries duration, have to be taken into account in order to develop the users' applications. 
The current challenge in mobile diabetes care is to combine several control strategies adapted to patient conditions and glycemic control state supported on communications and decision aid tools. The augmented reliability and availability of continuous glucose sensors, insulin pumps, mobile computing technologies and telemedicine services is making a reality the implementation of a feasible and reliable ambulatory closedloop glucose control system as the best current solution to achieve a good metabolic control for insulin-dependent diabetes mellitus patients. The research efforts on mobile technologies are moving in this direction looking for new solutions to better the quality of care and quality of life of people with diabetes.

\section{Acknowledgments}

This work has been partially funded by the Spanish FIS Projects PARIS (PI042466) and ADVISING (I060437). The authors wish to acknowledge the valuable work of all researchers from the Grupo de Bioingenieria y Telemedicina, specifically to V. Torralba.

\section{References}

1. International Diabetes Federation, Diabetes Atlas, $2^{\text {nd }}$ Edition (Brussels, 2003)

2. W.K. Waldhăusl, Finally we have arrived in a new millennium, Diabetologia 44,1 1. (2001)

3. A. de Leiva, P. Lefèbvre and I. Nerup, European dimension of Diabetes Research, Diabetologia 39, 5-11. (1995)

4. World Health Organization, Fact sheet $\mathrm{N}^{\circ} 312$, (September 2006)

5. S. Andreassen, E.J. Gómez and E.R. Carson, Computers in Diabetes 2000, Comput. Meth. Prog. Bioned. 62, 93-95. (2002)

6. D.G. Marrero, K.K. Kronz, M.P. Golden, Clinical evaluation of a computer-assisted self-monitoring of the blood glucose, Diabetes Care 12, 345-50. (1989)

7. K.K. Ahring, C. Joyce, J.P.K. Alring, N.R. Farid, Telephone modem access improves diabetes control in those with insulin-requiring diabetes, Diabetes Care 15, 971-5.(1992)

8. A. Billiard, V. Rohmer, M.A. Roques, M.G. Joseph, S. Suraniti, P. Girand, J.M. Limal, P. Fressinand and M. Marre, Telematic transmission of computerised blood glucose profiles for IDDM patients. Diabetes Care 14, 130-134. (1991)

9. R. Bellazzi, C. Larizza, S. Montani, A. Riva, M. Stefanelli, d'Annunzio, R. Lorini, E.J. Gómez, E. Hernando, E. Brugués, J. Cermeno, R. Corcoy, A. de Leiva, C. Cobelli, G. Nucci, S. Del Prato, A. Maran, E. Kilkki, J. Tuominen, A Telemedicine 
support for diabetes management: the T-IDDM project, Comp. Meth. and Prog. in Biomedicine 69, .147-162. (2002)

10. F. del Pozo, E.J. Gómez and M.T. Arredondo, A Telemedicine approach to Diabetes Management, Diab. Nutr. Metab. 4 (Suppl. 1), 149-153. (1991)

11. E.J. Gómez, F. del Pozo, and M.E. Hernando, Telemedicine for Diabetes Care: the DIABTel approach towards Diabetes Telecare, Med. Inform. 21(4), 283-295. (1996)

12. E.D. Lehmann, Application of information technology in clinical diabetes care. Part 1 Databases, algorithms and decision support, Med. Inform. 21(4), 255-374. (1996)

13. M.W. Tsang, M. Mok, G. Kam, M. Jung, A. Tang, U. Chan, C.M. Chu, I. Li and J. Chan, Improvement in diabetes control with a monitoring system based on a handheld, touch-screen electronic diary, J. of Telemedicine and Telecare 7(1) (2001)

14. M. Edmonds, M. Bauer, S. Osborn, H. Lutfiyya, J. Mahon, G. Doig, P. Grundy, C. Gittens, G. Molenkamp, D. Fenlon, Using the vista 350 telephone to communicate the results of home monitoring of diabetes mellitus to a central database and to provide feedback. Int. Journal of Medical Informatics 51(2). (1998)

15. D. J. Nigrin, I. S. Kohane, Glucoweb: A Case Study of Secure, Remote Biomonitoring and Communication. Proceedings of the AMIA 2000, (Los Angeles USA, November, 2000).

16. Dan Steinbock. The Mobile Revolution. The Making of Worldwide Mobile Markets. Published by Kogan Page (Business and Management). ISBN-10: 0749442964 (June 2005)

17. M.E. Hernando, E.J. Gomez, A. Garcia, and F. del Pozo, A multi-access server for the virtual management of diabetes, in Proc. ESEM 99 (Fifth conference of the European Society for Engineering and Medicine), 309-310. (1999)

18. American Diabetes Association, (2007) http://www.diabetes.org/

19. Report of the Expert Committee on the Diagnosis and Classification of Diabetes Mellitus, Diabetes Care, 25 (Suppl. 1), S5-S20, 2002.

20. The Diabetes Control and Complications Trial Research Group, The effect of intensive treatment of diabetes on the development and progression of long-term complications in insulin-dependent diabetes mellitus, N. Engl. J. Med. 329:977-986, 1993.

21. American Diabetes Association, 2003, Implications of the Diabetes Control and Complications Trial, Diabetes Care 26 (Suppl. 1), S25-S27.

22. American Diabetes Association, 2003, Standards of Medical Care for Patients with Diabetes Mellitus Diabetes Care 26 (Suppl. 1), S33-S50

23. A. García-Olaya, E.J. Gómez, M.E. Hernando, F. del Pozo, A Middleware CSCW Architecture for Diabetes Shared Care, IFMBE Proc. EMBEC'02, 3(2), 1376-1377. (2002)

24. B.W. Bode, T.M. Gross, K.R. Thornton, J. Mastrototaro. Continuous glucose monitoring used to adjust diabetes therapy improves glycosylated hemoglobin: a pilot study, Diabetes Research and clinical Practice, 46: 183-90, (1999).

25. R. Bellazzi, G. Nucci and C. Cobelli, The Subcutaneous Route to InsulinDependent Diabetes Therapy, IEEE Eng. Med. Biol. Magazine, 20 (1): 54-64, (2001) 
26. R.S. Parker, F.S. Doyle, and N. Peppas, The Intravenous Route to Blood Glucose Control- A review of Control Algorithms for Noninvasive Monitoring and Regulation in Type 1 Diabetic Patients, IEEE Eng. In Med. and Biol., 20(1): 65-73, (2001)

27. S.S. Chan, X. Fang, J. Brzezinski, Y. Zhou, S. Xu, J. Lam. Usability for mobile commerce across multiple form factors. Joumal of Electronic Commerce Research, Vol. $3(2002)$

28. J. Hobart. Designing Mobile Applications: Principles and Guidelines for Succesful UI Desing. http://www.classicsys.com/

29. Y. Chu, A. Ganz, Mobile Telemedicine Systems Using 3G Wireless Networks, Business Briefings: US Healthcare Strategies (2005)

30. MedicalGuard, https://www.medicalguard.net/modules.php?name=whereis

31. Think Positive Diabetes, https://www.thinkdiabetes.com/tpdiabetes/

32. Emminems, http://www.emminens.com/

33. GlucoMON, http://www.diabetech.net/glucomon.html

34. SiDiary, http://www. sidiary.org/

35. D. Gammon, E. Arsand, O.A. Walseth, N. Adersson, M. Jenssen and T. Taylor, Parent-Child Interaction Using a Mobile and Wireless System for Blood Glucose Monitoring, Journal of Medical Internet Research (November 2005)

36. A.J. Farmer, O.J. Gibson, C. Dudley, K. Bryden, P.M. Hayton, L. Tarassenko, A. Neil, A Randomized Controlled Trial of the Effect of Real-Time Telemedicine Support on Glycemic Control in Young Adults With Type 1 Diabetes, Diabetes Care, 28, 11, 2697-2702. (2005)

37. V. Franklin, A. Waller, C. Pagliari, S. Green, "Sweet Talk": Text Messaging Support for Intensive Insulin Therapy for Young People with Diabetes, Diabetes Technology \& Therapeutics, 5 6, 991-996 (2003)

38. E.J. Gómez, M.E. Hernando, T. Vering, M. Rigla, O. Bott, G. García-Sáez, P. Pretschner, E. Brugués, O. Schnell, C. Patte, J. Bergmann, R. Dudde and A. de Leiva, The INCA system: a further step towards a Telemedical Artificial Pancreas. IEEE Transaction on In formations Technology in Biomedicine (2007)

39. M. Rigla, M.E. Hernando, E.J. Gómez, E. Brugués, G. Garcia-Sáez, V. Torralba, A. Prados, L. Erdozain, J. Vilaverde and A. de Leiva, A Telemedicine System that Includes a Personal Assistant Improves Glycemic Control in Pump Treated Patients with Type 1 Diabetes. Journal of Diabetes Science and Technology. 1, 4, (2007) 